\title{
Pressure hull analysis under shock loading
}

\author{
Ya-Jung Lee ${ }^{\mathrm{a}}$, Chia-Hao Hsu ${ }^{\mathrm{b}, *}$ and Chien-Hua Huang ${ }^{\mathrm{a}}$ \\ ${ }^{a}$ National Taiwan University, Ship Technology Research Center, No. 1, Sec. 4, Roosevelt Road, Taipei, Taiwan \\ ${ }^{\mathrm{b}}$ United Ship Design and Development Center, 14F, 27, Sec. 2, Chung-cheng E. Road, Tamshui, Taipei, Taiwan
}

Received 27 March 2006

Revised 8 November 2006

\begin{abstract}
The hull of high performance submarines must resist underwater shock loading due to exploding torpedoes or depth bombs. An underwater shock involving an initial shock wave and successive bubble pulsating waves is so complex that a theoretical technique for deriving shock pressure distribution is required for improving simulation efficiency. Complete shock loading is obtained theoretically in this work, and responses of a submarine pressure hull are calculated using ABAQUS USA (Underwater Shock Analysis) codes. In the long run, this deflection and stress data will assist in examining the structural arrangement of the submarine pressure hull.
\end{abstract}

Keywords: Submarine, pressure hull, underwater shock

\section{Introduction}

A typical submarine structure system can be deconstructed into four parts: outer shell, pressure hull, sail, and rudder. The pressure hull is typically composed from fore to aft of three conventional pressure hulls: dome, cylinder, and cone. Pressure hulls must resist high water pressure to protect humans and instruments and, thus, require critical strength. During attack, antisubmarine weapons, such as depth bombs and torpedoes, generate powerful explosive forces that can seriously damage a submarine's structure. This work focuses on pressure-hull strength, an important component of a submarine's structural design, under shock loading of the submerged submarine.

When an underwater explosion occurs, the explosive charge in the bomb is rapidly converted into a gas at a very high temperature and pressure. An explosion's initial shock wave is followed by a succession of pressure waves at $10-15 \%$ of the initial shock-wave pressure. These waves result from repeated expanding and contracting of the high-pressure gas. The initial shock wave, which has a short duration and a high-pressure variation, can be considered as an extremely brief transient load with respect to its action on a vessel. Conversely, although the shock waves resulting from the bubble pulses are substantially smaller than the initial shock wave, they typically last longer, and result in vibratory submarine motion, since the duration of a bubble-pulse cycle can be close to the fundamental vibration period of a ship. In this work the initial shock wave and bubble-pulsating waves are applied as the total load during underwater shock analysis.

Although finite element method (FEM) packages, such as ABAQUS with an Underwater Shock Analysis (USA) module, can solve for the explosive load - including the initial shock wave and bubble-pulsating waves - massive computing resources are required. This work attempts to obtain theoretically the total incident pressure, and uses this pressure as the input for ABAQUS. Figure 1 presents the methodology flow chart used to solve the underwater shock problem. An initial shock wave (see left-hand column of Fig. 1) can be defined effectively using empirical equations from the literature; however, the bubble-pulsating waves must be extracted from a series of theories (see right-hand column). Then ABAQUS software is utilized for computations. Each theory portrayed in Fig. 1 is described separately in this paper.

This work utilizes the pressure-hull model that simulates the German HDW U209-1400 submarine (Fig. 2). Analysis results are used to evaluate the submarine's ability to resist underwater explosions.

\footnotetext{
*Corresponding author. Tel.: +886 2 28085899; Fax: +886 2 28085866; E-mail: jeffsheu @ mail.usddc.org.tw.
} 


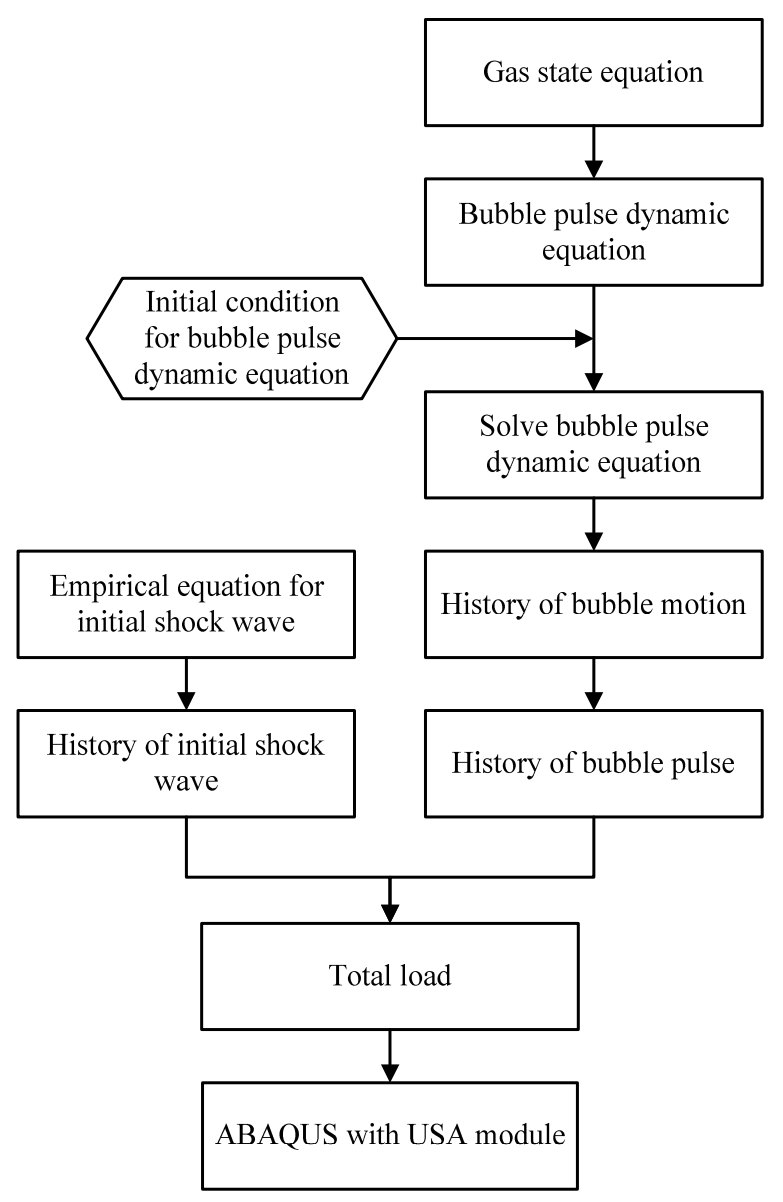

Fig. 1. Solution methodology.

\section{Empirical equation for initial shock wave}

The initial underwater shock wave can be approximated using an exponential function that describes the pressure history of a shock wave as observed from fixed location. The shock wave comprises an initial, instantaneous increase in pressure, followed by an exponential decline. This equation is well known as Cole's empirical equation [1]:

$$
P_{s w}(R, t, W)=P_{\max }(R, W) \cdot e^{\frac{-t}{\theta_{e}(R, W)}},
$$

where $P_{s w}(R, t, W)$ is the shock-wave pressure at the observation point, $R$ is the distance between observation point and explosion center $(\mathrm{m}), W$ is the weight of explosive charge $(\mathrm{kg}), P_{\max }(R, W)$ is the maximum pressure (Pa); $t$ is the time elapsed since the arrival of the shock; and $\theta_{e}(R, W)$ is the decay time constant, which is the time of observed pressure decays from $P_{\max }$ to $P_{\max } / e$. Peak pressure $P_{\max }$ and decay constant $\theta_{e}$ are depicted by Eqs (2) and (3), respectively:

$$
P_{\max }(R, W)=\alpha_{1}\left(\frac{W^{1 / 3}}{R}\right)^{\beta_{1}}
$$

and

$$
\theta_{e}(R, W)=\alpha_{2} \cdot W^{1 / 3}\left(\frac{W^{1 / 3}}{R}\right)^{\beta_{2}}
$$

where $\alpha_{1}, \beta_{1}, \alpha_{2}$ and $\beta_{2}$ are constants based on the explosive charge type. For a TNT charge, $\alpha_{1}=5.212 \times 10^{7}$, $\beta_{1}=1.18, \alpha_{2}=0.0895 \times 10^{-3}$ and $\beta_{2}=-0.185$. 


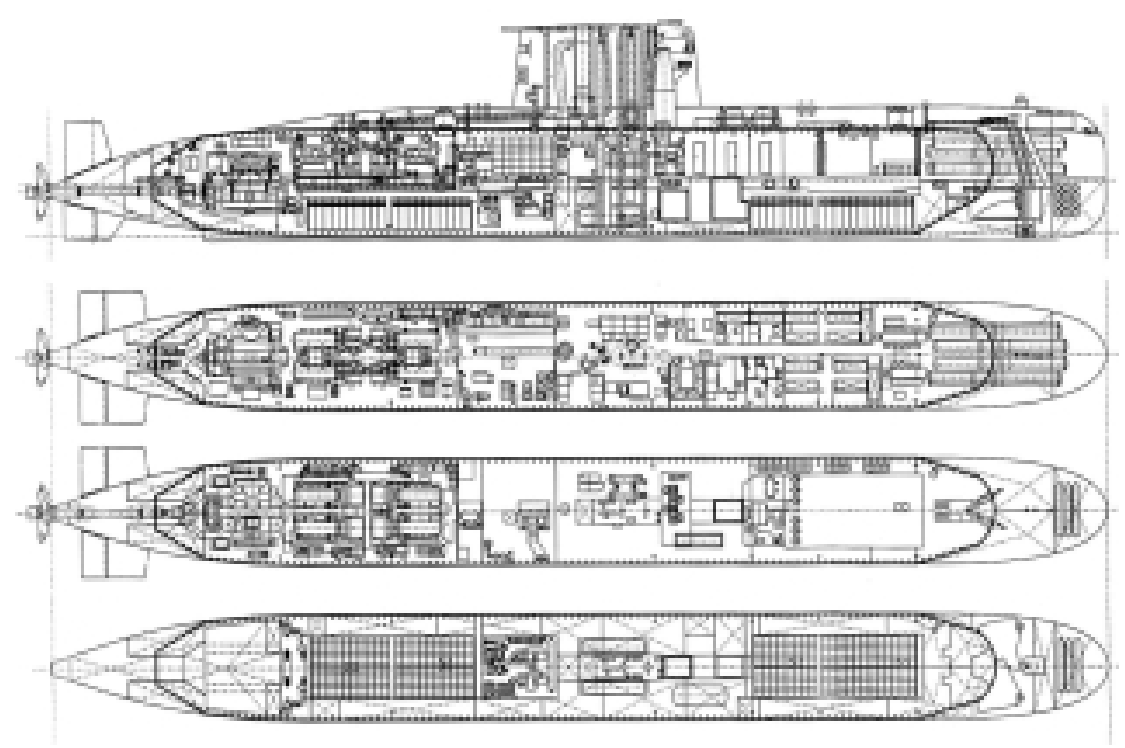

Fig. 2. General arrangement of the U209-1400 Submarine.

\section{Bubble-pulse dynamic equation}

The bubble-pulse dynamic model can be addressed using two principal approaches: considering the surrounding fluid as non-compressible, or as compressible. In 1917, Rayleigh [2] constructed a dynamic energy equation for an expanding bubble in a boundless incompressible field. In 1923, Lamb [3] developed a dynamic bubble model under an adiabatic expansion condition and derived a bubble-pulse dynamic equation for an incompressible fluid field; this model is known as the Rayleigh-Lamb dynamic equation. The complete bubble-pulse dynamic equation, also named the Doubly Asymptotic Equation of Motion, can be further derived based on the Rayleigh-Lamb dynamic equation:

$$
a \ddot{a}+\frac{3}{2} \dot{a}^{2}=\rho^{-1}\left\{\left[1+\left(\frac{a}{c}\right) \frac{\mathrm{d}}{\mathrm{dt}}\right] P_{g}-P_{h}\right\}
$$

where $a$ is the bubble radius, $P_{g}$ is the bubble interior pressure, $P_{h}=\rho g H_{0}$ (static water pressure at the bubble center $H_{0}$ ), and $c$ is the sound speed in the fluid. Solving Eq. (4) yields the bubble radius history, where the interior bubble pressure $P_{g}$ comes from the gas state equation (discussed in the following section).

\section{Gas state equation}

The equation describing the gas pressure variation in the bubble following an explosion is called the gas state equation. In 1948, Jones and Miller [4] utilized the assumption of heat- and chemical energy conservation for an entire explosion to develop the Virial Expansion theory that yields the pressure of bursting gas. Later, in 1950, Taylor [5] applied this theory and, assuming that the specific heat ratio $\gamma_{g}$ is constant during an explosion, obtained the Jones-Miller-Taylor Equation of State (JMT EOS):

$$
P_{g}=K\left(\frac{W}{V_{g}}\right)^{\gamma_{g}}
$$

where $K$ is a parameter varying with dynamite type and charge density, $W$ is the charge weight, and $V_{g}$ is the bubble volume. Table 1 defines the parameters for a TNT charge. 
Table 1

Parameters for TNT

\begin{tabular}{ccc}
\hline Charge density $\left(\mathrm{kg} / \mathrm{m}^{3}\right)$ & $K \times 10^{-3}\left(\mathrm{~Pa} \cdot\left(\mathrm{m}^{3} / \mathrm{kg}\right)^{\gamma g}\right)$ & $\gamma_{g}$ \\
\hline 1000 & 139.7 & 1.20 \\
1500 & 77.7 & 1.27 \\
1630 & 69.71 & 1.30 \\
\hline
\end{tabular}

\section{Initial condition for the bubble pulse dynamic equation}

To solve the bubble-pulse dynamic equation, Eq. (4), appropriate initial conditions, including initial bubble radius $a(0)$ and explosion speed $\dot{a}(0)$, are needed. In ideal conditions, assume that a charge explodes and transforms into a high-temperature, high-pressure gas with the same mass and volume as that of its original solid state. The gas then pierces the packing shell of the dynamite and explodes into the surrounding water. In this case, $a(0)=a_{c}\left(a_{c}\right.$ is the charge's radius) and $\dot{a}(0)=0$.

However, the bubble-pulse dynamic equation cannot effectively describe an explosion at the initial explosion time due to the complexity of the energy conversion event. Hunter [6] proposed utilizing the condition at the time ranging between $3 \theta_{e}$ and $7 \theta_{e}$ as the initial condition.

In 1950, J.S. Coles [1] used experimental results to modify Eq. (1) to

$$
P_{s w}(R, t, W)=P_{\max }(R, W) \cdot\left\{e^{-\frac{t^{*}}{\theta_{e}(R, W)}}+0.01\left[\frac{t^{*}}{\theta_{e}(R, W)}\right]^{3} e^{-\frac{t^{*}}{1.65 \theta_{e}(R, W)}}\right\} .
$$

The applicable time range in Eq. (6) is the time when the initial condition is given, which can be set as $3 \theta_{e}$ to $7 \theta_{e}$. ( $t^{*}$ is the time when the initial condition is given).

In 1956, Strasberg [7] surveyed acoustic theory for a spherical surface with large movement and solved the relationship between the bubble radius and the shock pressure as

$$
\dot{a}\left(t^{*}\right)=\frac{R P_{\max }}{\rho a\left(t^{*}\right)}\left\{\theta_{e}\left(1+0.06 b^{4}\right)-\theta_{e} e^{\frac{-t^{*}}{\theta_{e}}}-\left(0.06 b^{4} \theta_{e}+0.06 b^{3} t^{*}+0.03 \frac{b^{2} t^{* 2}}{\theta_{e}}+0.01 \frac{b t^{* 3}}{\theta_{e}^{2}}\right) e^{\frac{-t^{*}}{\theta_{e}}}\right\}
$$

and

$$
\begin{aligned}
a^{3}\left(t^{*}\right)= & \left\{a_{c}^{3}+\frac{3 R P_{\max }}{\rho}\left[\theta_{e}\left(1+\theta_{e} e^{\frac{-t^{*}}{\theta_{e}}}\right) t^{*}+0.06 b^{4} \theta_{e} t^{*}\right.\right. \\
& \left.\left.-\left(0.24 b^{5} \theta_{e}^{2}+0.18 b^{4} \theta_{e} t^{*}+0.06 b^{3} t^{* 2}+0.01 \frac{b^{2} t^{* 3}}{\theta_{e}}\right) e^{\frac{-t^{*}}{b \theta_{e}}}\right]\right\} .
\end{aligned}
$$

Equation (6) yields maximum pressure $P_{\max }$. Substituting $P_{\max }$ into Eqs (7) and (8) produces the initial conditions $a\left(t^{*}\right)$ and $\dot{a}\left(t^{*}\right)$.

\section{Empirical formula for bubble dynamics [8]}

For TNT charges, an approximation of the maximum bubble radius $\left(a_{\max }\right)$ and the first period of the bubble-pulse wave $\left(T_{1}\right)$ can be expressed using the empirical formulae below in Eqs (9) and (10):

$$
a_{\max }=1.6\left(\frac{W}{D+10.34}\right)^{\frac{1}{3}}(\mathrm{~m})
$$

and

$$
T_{1}=0.985 \frac{W^{1 / 3}}{(D+10.34)^{5 / 6}}(\mathrm{sec}) .
$$

$W$ is the weight of the charge in newtons, and $D$ is the depth of an explosion in meters. These two empirical equations can be used for a comparison after extracting the bubble motion histories theoretically. 


\section{Pressure variation caused by bubble motion}

To allocate pressure variation caused by bubble motion $a(t)$, assumptions are required to simplify the physical phenomenon. The liquid density $\rho_{L}$ and the dynamic viscosity $\mu_{L}$ are assumed constant; and the contents of the bubble are assumed homogeneous, that is, to have uniform temperature $T_{B}(t)$ and pressure $P_{B}(t)$ [9].

Outside the bubble, $P_{b u}(R, t)$ and $T(R, t)$ denote pressure and temperature respectively; and $u(R, t)$ denotes the radial velocity outward for the bubble. Conservation of mass requires that

$$
u(R, t)=\frac{F(t)}{R^{2}},
$$

where $F(t)$ is the kinematic boundary condition at the bubble surface. In the ideal case of zero mass crossing the bubble surface, it is clear that $u(a, t)=\mathrm{d} a / \mathrm{dt}$; hence,

$$
F(t)=a^{2} \frac{\mathrm{d} a}{\mathrm{dt}}
$$

Equation (12) is typically an effective boundary denotation, even when considering evaporation and condensation of a fluid occurring continually on the interface. Newly produced vapor volume must equal bubble volume variation, that is, $4 \pi a^{2} \cdot \mathrm{d} a / \mathrm{dt}$; therefore, the mass flow rate of evaporation is

$$
\rho_{V}\left(T_{B}\right) \cdot 4 \pi a^{2} \cdot \frac{\mathrm{d} a}{\mathrm{dt}}
$$

where $\rho_{V}\left(T_{B}\right)$ is the saturated vapor density at bubble temperature $T_{B}$. Expression (13) must equal the mass flow rate of liquid moving inward to the interface and, hence, the inward velocity of liquid can be obtained as $\rho_{V}\left(T_{B}\right) \cdot \frac{\mathrm{d} a}{\mathrm{dt}} / \rho_{L}$. Equations (11) and (12) are then further expanded into Eqs (14) and (15):

$$
\begin{aligned}
& u(a, t)=\frac{\mathrm{d} a}{\mathrm{dt}}-\frac{\rho_{V}\left(T_{B}\right)}{\rho_{L}} \cdot \frac{\mathrm{d} a}{\mathrm{dt}}=\left[1-\frac{\rho_{V}\left(T_{B}\right)}{\rho_{L}}\right] \cdot \frac{\mathrm{d} a}{\mathrm{dt}}, \\
& F(t)=\left[1-\frac{\rho_{V}\left(T_{B}\right)}{\rho_{L}}\right] \cdot a^{2} \cdot \frac{\mathrm{d} a}{\mathrm{dt}} .
\end{aligned}
$$

In practical cases, $\rho_{V}\left(T_{B}\right)<<\rho_{L}$ and, therefore, the approximate form of Eq. (11) is sufficient and will be employed hereafter.

Assuming seawater is a newtonian liquid, the Navier-Stokes equation for motion in the $R$ direction is

$$
-\frac{1}{\rho_{L}} \frac{\partial P_{b u}}{\partial R}=\frac{\partial u}{\partial t}+u \frac{\partial u}{\partial R}-\mu_{L}\left[\frac{1}{R^{2}} \frac{\partial}{\partial R}\left(R^{2} \frac{\partial u}{\partial R}\right)-\frac{2 u}{R^{2}}\right] .
$$

Substituting the expression for $u$ from Eq. (11) generates

$$
-\frac{1}{\rho_{L}} \frac{\partial P_{b u}}{\partial R}=\frac{1}{R^{2}} \frac{\mathrm{d} F}{\mathrm{dt}}-\frac{2 F^{2}}{R^{5}} .
$$

Upon integrating Eq. (17), one obtains:

$$
\frac{P_{b u}-P_{\infty}}{\rho_{L}}=\frac{1}{R} \frac{\mathrm{d} F}{\mathrm{dt}}-\frac{F^{2}}{2 R^{4}},
$$

where $P_{\infty}$ is the pressure far from the explosion; this is commonly the static water pressure at explosion depth.

Finally, an equation for water pressure variation caused by bubble pulsating motion can be derived using the condition $P_{b u} \rightarrow P_{\infty}$ while $R \rightarrow R_{\infty}$ :

$$
\begin{aligned}
P_{b u}(R, t) & =P_{\infty}+\rho_{L} \cdot\left\{\frac{1}{R}\left[2 a \cdot\left(\frac{\mathrm{d} a}{\mathrm{dt}}\right)^{2}+a^{2} \cdot \frac{\mathrm{d}^{2} a}{\mathrm{dt}^{2}}\right]-\frac{a^{4}}{2 R^{4}} \cdot\left(\frac{\mathrm{d} a}{\mathrm{dt}}\right)^{2}\right\} \\
& =P_{\infty}+\rho_{L} \cdot\left[\frac{1}{R}\left(2 a \cdot \dot{a}^{2}+a^{2} \cdot \ddot{a}\right)-\frac{a^{4}}{2 R^{4}} \cdot \dot{a}^{2}\right] .
\end{aligned}
$$




\section{The fluid-structure interaction effects}

Suppose a condition of a given structure (e.g., a submarine or ship) submerged or semi-submerged in water; the water, as an ideal medium, should transmit linear wave motion [10]. When $P_{i}$ is the incident wave, the diffracted wave due to a rigid body is $P_{d}$ and the reflected wave from the water surface is $P_{p}$; the pressure field in the water is represented by Eq. (20):

$$
P=P_{i}+P_{d}+P_{p} \text {. }
$$

On the wetted surface $\left(S_{0}\right)$ of a ship, the boundary condition is

$$
\frac{\partial P}{\partial n}=-\rho \ddot{u}_{n}
$$

where $n$ is the normal unit vector on the structure surface, $\rho$ is the fluid density, $u$ is the surface displacement of the structure and $\ddot{u}_{n}$ is the normal acceleration of the structure surface.

At the water surface $S_{p}$, the characteristic impedances of air and water vary significantly, such that the pressure wave cannot pass through and, therefore, is regarded as zero:

$$
P=0 \text { on } S_{p} \text {. }
$$

At infinity, the following Sommerfeld radiation condition should be satisfied:

$$
\lim _{r \rightarrow \infty} r\left[\frac{\partial P}{\partial n}+i k P\right]=0,
$$

where $r$ is the distance between the observation point and explosion center, $i=\sqrt{-1}$, and $k$ is the wave number.

Furthermore, the wave motion should satisfy the following Helmholtz equation:

$$
\nabla^{2} \phi=\frac{1}{c^{2}} \frac{\partial^{2} P}{\partial t^{2}}
$$

where $c$ is sound speed, and $\nabla^{2}$ is the Laplacian operator.

In this work, the incident wave $P_{i}$ comprises the initial shock wave and bubble pulsating wave, and can be configured as

$$
P_{i}=P_{s w}(R, t, W)+P_{b u}(R, t) .
$$

\section{Coupled fluid-structure interaction equations [11]}

To solve the coupled fluid-structure interaction problems, the FEM software ABAQUS combined with the USA module, which is theoretically based on the Doubly Asymptotic Approximations (DAA), is utilized. During the solution process, the USA structural interface (USI) elements should be constructed on the structure surface that is connected with the ship structure and DAA boundary. The incident wave spreads to the DAA boundary, and the USA transforms the wave into pressure acting on the structure surface. Next, ABAQUS performs structure transient analysis and USA solves the coupled fluid-structure interaction equations based on displacement and velocity of nodes.

The coupled fluid-structure interaction equations are

$$
\begin{aligned}
& {\left[M_{f}\right]\left\{\ddot{q}_{s}\right\}+\rho_{f} c\left[A_{f}\right]\left\{\dot{q}_{s}\right\}+\rho_{f} c\left[\Omega_{f}\right]\left[A_{f}\right]\left\{q_{s}\right\}=} \\
& \rho_{f} c\left[\left[M_{f}\right]\left([G]^{T}\{\ddot{u}\}-\left\{\dot{u}_{I}\right\}\right)+\left[\Omega_{f}\right]\left[M_{f}\right]\left([G]^{T}\{\dot{u}\}-\left\{u_{I}\right\}\right)\right]
\end{aligned}
$$

and

$$
\left[M_{s}\right]\{\ddot{u}\}+\left[C_{s}\right]\{\dot{u}\}+\left[K_{s}\right]\{u\}=-[G]\left[A_{f}\right]\{P\},
$$

where $\left\{q_{s}\right\}=\int_{0}^{t}\left\{u_{s}(\tau)\right\} d \tau,\left[M_{f}\right]$ is the symmetrical fluid mass matrix, $\rho_{f}$ is the fluid density, $\left[A_{f}\right]$ is the diagonal matrix of fluid areas, $[G]^{T}$ transforms the nodal structural velocities into normal surface velocities at the centroid of each fluid element, $\left[M_{s}\right]$ is the structure mass matrix, $\left[C_{s}\right]$ is the damping matrix, and $\left[K_{s}\right]$ is the stiffness matrix for the structure and $\Omega_{f}=\eta \rho_{f} c A M_{f}^{-1}$ when the scalar parameter is bounded as $0 \leqslant \eta \leqslant 1$. 
Table 2

Principal specifications of the U209

\begin{tabular}{ll}
\hline Length & $62 \mathrm{~m}$ \\
\hline Diameter of pressure hull & $6.2 \mathrm{~m}$ \\
Floating displacement & $1454 \mathrm{ton}$ \\
Diving displacement & $1586 \mathrm{ton}$ \\
Operational diving depth & $250 \mathrm{~m}$ \\
BHP & $2800 \mathrm{~kW}$ \\
Maximum velocity in diving & $23 \mathrm{knot}$ \\
\hline
\end{tabular}

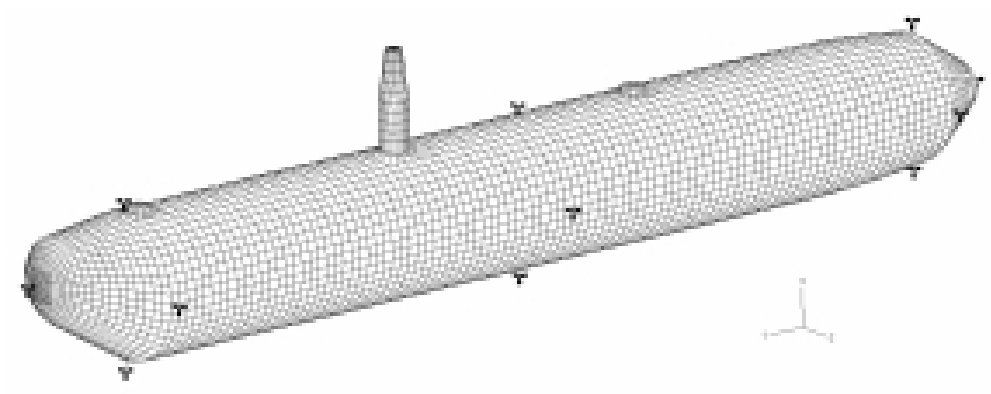

Fig. 3. Pressure hull model.

\section{Model for analysis}

The pressure hull model was adopted for the Germany HDW U209-1400. A typical U209 submarine is constructed with a single pressure hull composed of HY80 high-tensile steel. The U209 nominally carries 8 torpedo launch pads and 14 torpedoes; some U209s can launch anti-vessel missiles. Table 2 lists the principal specifications of the U209. Figure 3 presents the finite element model of the pressure hull constructed for this research. The black triads in Fig. 3 are damping boundaries that will be described in Section 13. A conductor tower and two access hatches are included; but the outer shell is omitted, as it does not resist water pressure. Although detailed structural configurations of the U209 cannot be obtained, the pressure hull dimensions including the outside surface, ribs and other stiffeners, were carefully constructed to represent the actuality as closely as possible (Fig. 2). The model has the capability of diving to $400 \mathrm{~m}$ based on an analysis considering geometrical nonlinearity and plastic material properties.

\section{Depth bomb}

Assume that a depth bomb, the explosion source, has TNT charges weighing $160 \mathrm{~kg}$ and a radius of $a_{c}=0.1625 \mathrm{~m}$. The TNT charge density is $1500 \mathrm{~kg} / \mathrm{m}^{3}$, with parameters $K=77.7 \mathrm{E}+3$, and $\gamma_{g}=1.27$ in Eq. (6). This bomb is exploded at $15.81 \mathrm{~m}$ beside the submarine hull while the submarine is at a depth of $250 \mathrm{~m}$. In this scenario, the Keel Shock Factor (KSF) is 0.8 , and conforms to the naval vessel design standard. The KSF, which indicates the degree of shock, is defined with Eq. (28):

$$
K S F=\frac{\sqrt{W}}{L}\left(\frac{1+\cos \theta}{2}\right),
$$

where $W$ is the charge weight, $L$ is the distance between explosion center and ship keel, and $\theta$ is the angle between the explosion center, free surface and ship Referring to Fig. $4, \theta_{S U B}$ is employed here for a submarine while $\theta_{S H I P}$ is adopted for a surface ship. 


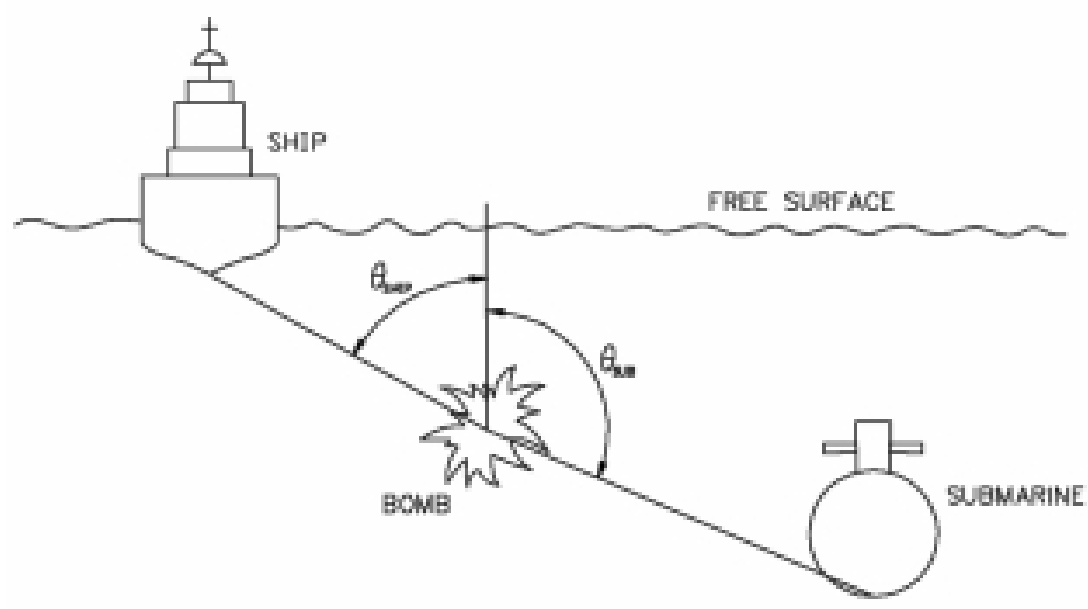

Fig. 4. Diagram for $\theta$ used in KSF calculation.

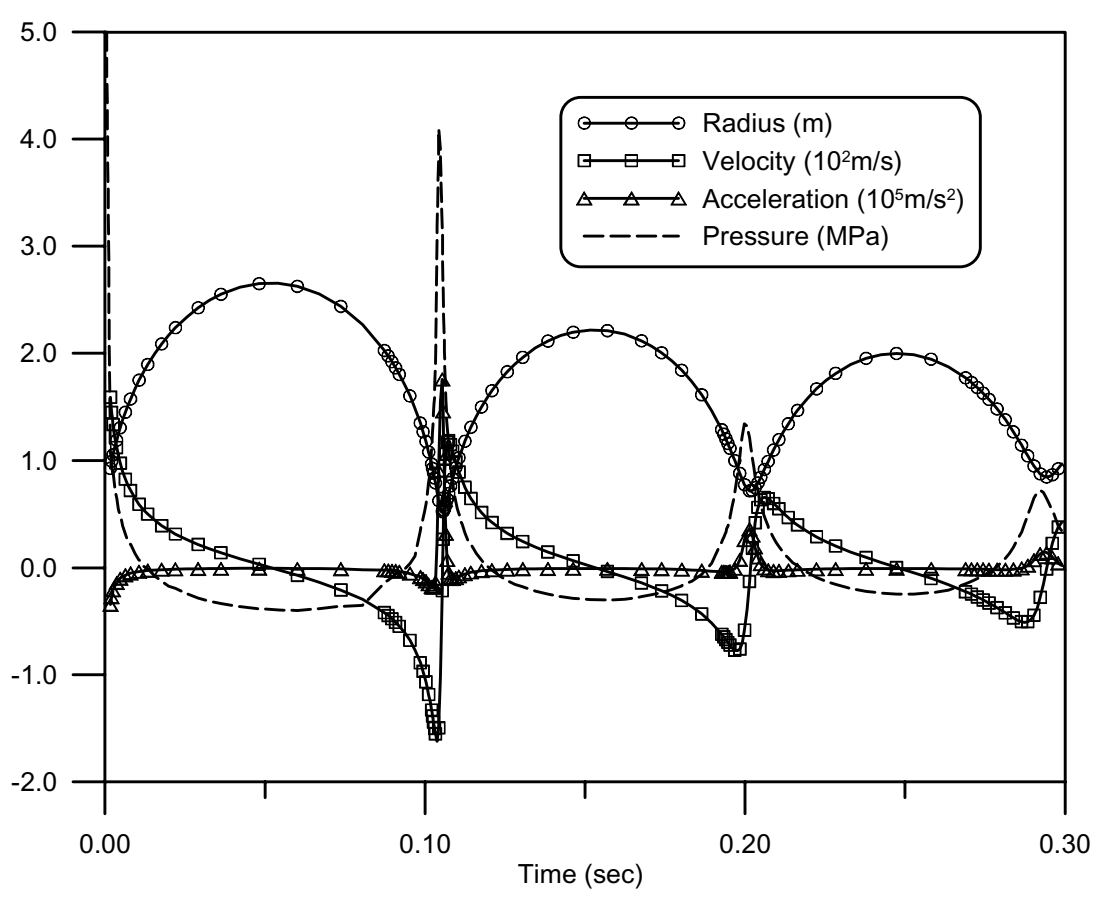

Fig. 5. Bubble motion history.

\section{Results of shock wave evaluation}

The histories of bubble motion and pressure variation for the simulated depth bomb are obtained theoretically (Fig. 5) and by using Eqs (4) and (19), which include the bubble radius, the swelling/contracting velocity, and the acceleration and pressure variation observed at the standoff point - a standoff point is the nearest point to the explosion center on the submarine surface. In Fig. 5, the time origin is the time at which the pressure wave first contacts the submarine surface. Simulation results indicate that the maximum shock pressure reaches $1.6034 \mathrm{e}+7 \mathrm{~Pa}$ 


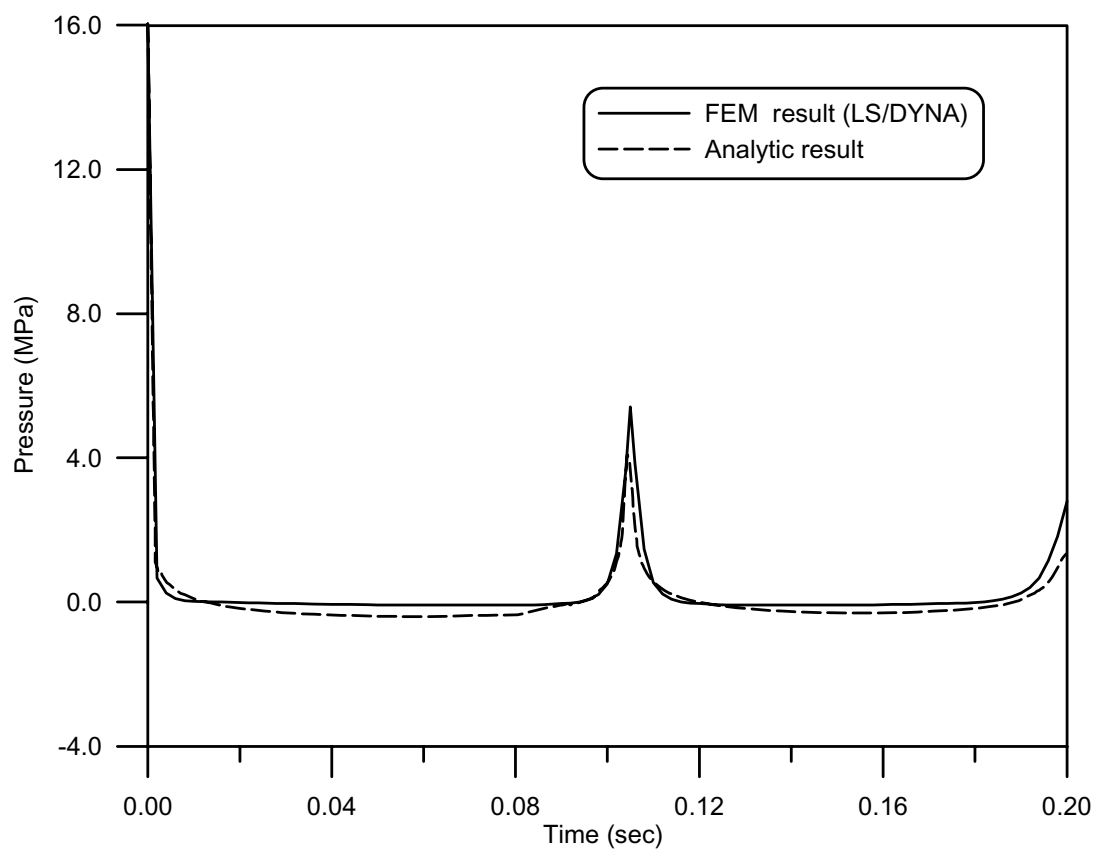

Fig. 6. Comparison of LS/DYNA and theoretical results.

and then declines immediately. Very small negative pressure (suction) also exists sometimes. At $t=1.055 \mathrm{e}-1 \mathrm{sec}$, the bubble contracts significantly to a minimum radius, followed by high-acceleration swelling that induces another shock wave similar to an explosion.

The empirical values of maximum bubble radius and first period of the bubble pulsating wave, derived by Eqs (9) and (10), are $a_{\max }=2.9 \mathrm{~m}$ and $T_{1}=0.1111 \mathrm{sec}$, respectively. The theoretical results for maximum bubble radius and first period of the bubble pulsating wave are $a_{\max }=2.65 \mathrm{~m}$ and $T_{1}=0.1055 \mathrm{sec}$, respectively. The closeness of these two results demonstrates the accuracy of the strategy applied in this work.

Additionally, the LS/DYNA commercial software is used to solve the Jones-Wilkins-Lee Equation of State (JWL EOS) using the same bomb and exploding position settings. Figure 6 presents the history of shock pressure variation compared with theoretical results. Maximum pressure $P_{\max }(5.0 \mathrm{MPa})$ calculated by LS/DYNA is higher than that (4.0 MPa) calculated using the theoretical approach, although both peaks occur at almost the same time. The difference between two maximum pressures is thought to be due to the fact that the $P_{\max }$ derived by Eq. (19) required the data for bubble radii $a, \dot{a}$ and $\ddot{a}$, which were obtained from Eqs (4), (7) and (8). The bubble radius formulae were derived from integration procedures that likely accumulate errors and lead to a smaller bubble radius and, consequently, a smaller pressure than the LS/DYNA's results.

\section{Damping boundary condition for pressure hull}

Some clamped boundary conditions for a submarine hull are incorrect for an underwater shock problem. Submarines vibrate and move slightly during a shock wave. Moreover, the fluid surrounding the submarine has added mass and damping effects. In this work, the added water mass is addressed by the ABAQUS USA; however, the damping forces acting on the submarine must be determined.

The total damping coefficient in the heave direction, $C_{H D}$ [12], can be defined by

$$
C_{H D}=\frac{1}{2} \rho B^{2} U K_{L},
$$

where $\rho$ is the fluid density, $B$ is the ship's width, $U$ is velocity, and $K_{L}$ is the first-order differentiation of the $C_{L}-\alpha$ chart (chart of lift coefficient vs. angle of attack for a submarine cross section). 


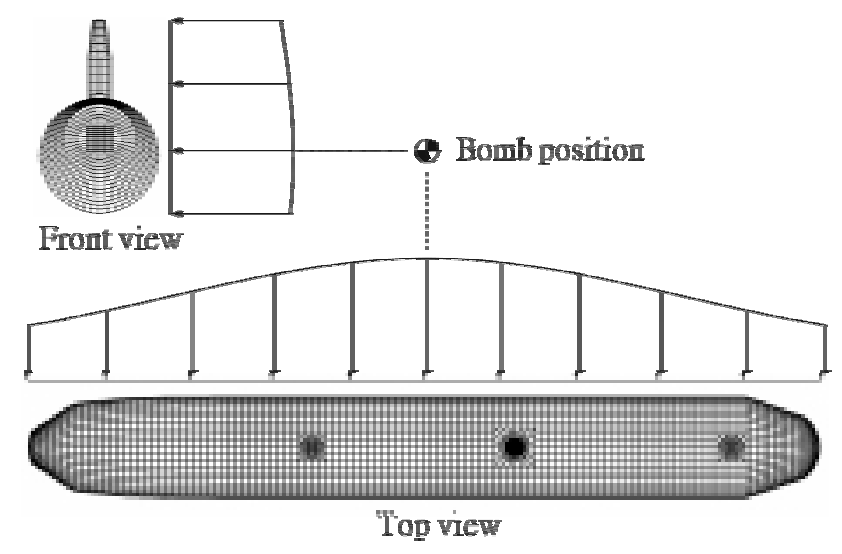

Fig. 7. Bomb position and pressure distribution.

Calculate $C_{H D}$ utilizing the empirically estimated velocity of the submarine subjected to a specific load. This work assumes that damping forces are distributed uniformly on the submarine surface. Total damping forces are simulated using 14 sets of connector elements with damping. These connector elements are placed on three hull sections (front, middle, and back sections) with four groups for each section; the remaining two groups are on the bow and stern. Each group contains three connectors that are pointed toward three space coordinate directions. The damping coefficients (force per relative velocity, $\mathrm{N} \cdot \mathrm{sec} / \mathrm{m}$ ) can be reasonably specified in the range $10^{2}-10^{3}$; here, the value employed is $10^{3}$. One end of a connector is attached to the hull and the other is fixed in space. This arrangement facilitates finite movement of the pressure hull. The black triads in Fig. 3 present the damping connector locations.

Furthermore, based on preliminary calculated results, when stress is concentrated on the connected point, more numbers of connector can be utilized to substitute one connector while decreasing the damping coefficient for each connector.

\section{Results of hull response}

In Fig. 7, the target point indicates the bomb positioned beside the hull, and the arrows indicate shock wave pressure distribution.

According to calculated response results (Figs 8 and 9), the submarine first deforms and shifts in the force direction. At $t=0.005 \mathrm{sec}$, the instantaneous acceleration is $650 \mathrm{G}$. As the shock wave decays, acceleration decreases; however, at $t=0.11 \mathrm{sec}$, acceleration again peaks at $-250 \mathrm{G}$ due to the bubble pulse.

Next, the maximum stress $(420 \mathrm{MPa})$ at the standoff point occurs at $t=0.02 \mathrm{sec}$, at which time the region surrounding the standoff point also attains maximum displacement. Computational results show that the front and back extremities of the hull deform less than the standoff point, which is located at the middle hull. At $t=0.07 \mathrm{sec}$, the displacement is in the opposite direction: the two extremities have larger displacements than the standoff point, implying that the fundamental-mode vibration period for this hull is $0.1 \mathrm{sec}$, a short period.

Stress and displacement are described as follows. When $t=0.0 \mathrm{sec}$, the shock wave has not yet contacted the hull and, thus, stresses and displacements are zero. When $t=0.01 \mathrm{sec}$ (Figs 10 and 11), the pressure hull is affected by the initial shock wave, inducing an elevating stress (590 MPa) near the forward access hatch and yielding the local hull. When $t=0.04 \mathrm{sec}$ (Fig. 12), the stress concentrates at the bottom of conductor tower and the aft access hatch.

When $t=0.12 \mathrm{sec}$ (Fig. 13), with the bubble pulsating wave passing through the hull, stress reaches $575 \mathrm{MPa}$, causing the plates at the conductor tower bottom to yield. Finally, at $t=0.2 \mathrm{sec}$ (Figs 14 and 15), the stress becomes low and the next pulsating bubble wave comes into play. At this time, total shift and deformation at the midship section is $24 \mathrm{~cm}$; and at the stern, $27 \mathrm{~cm}$. 


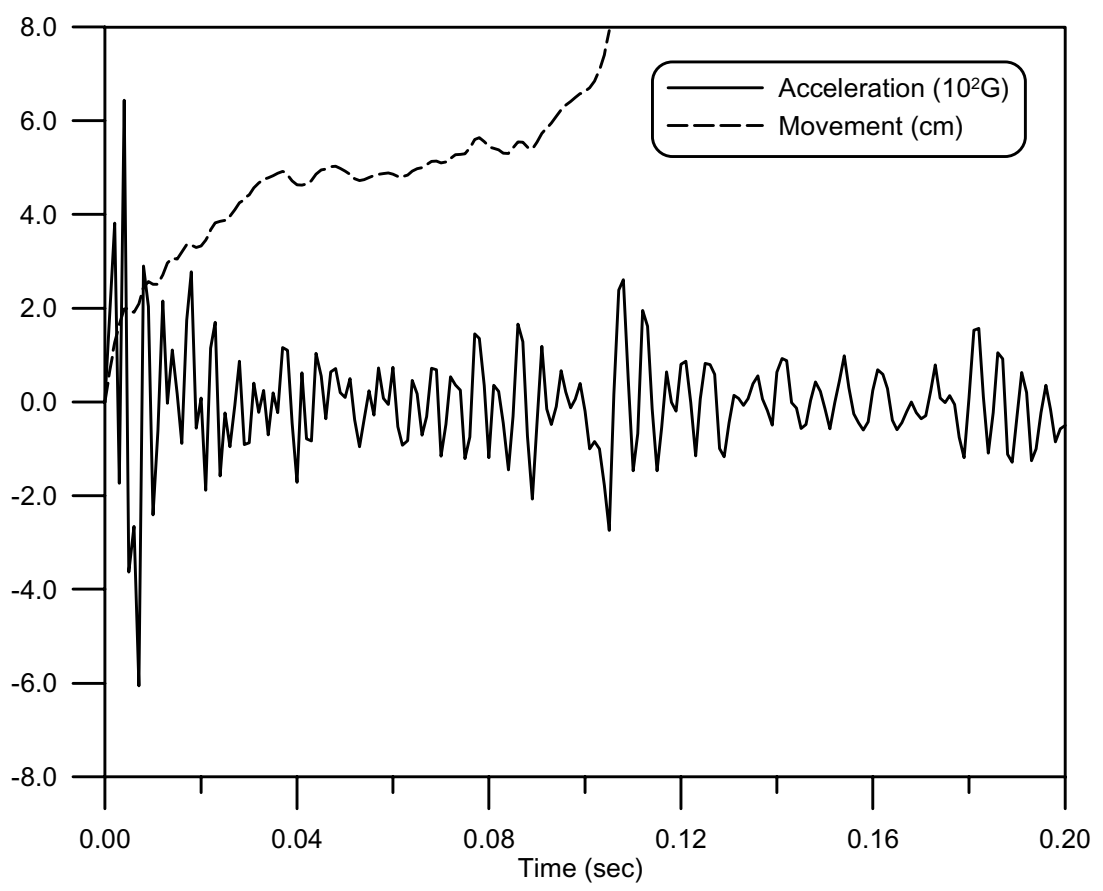

Fig. 8. Acceleration and movement at the standoff point.

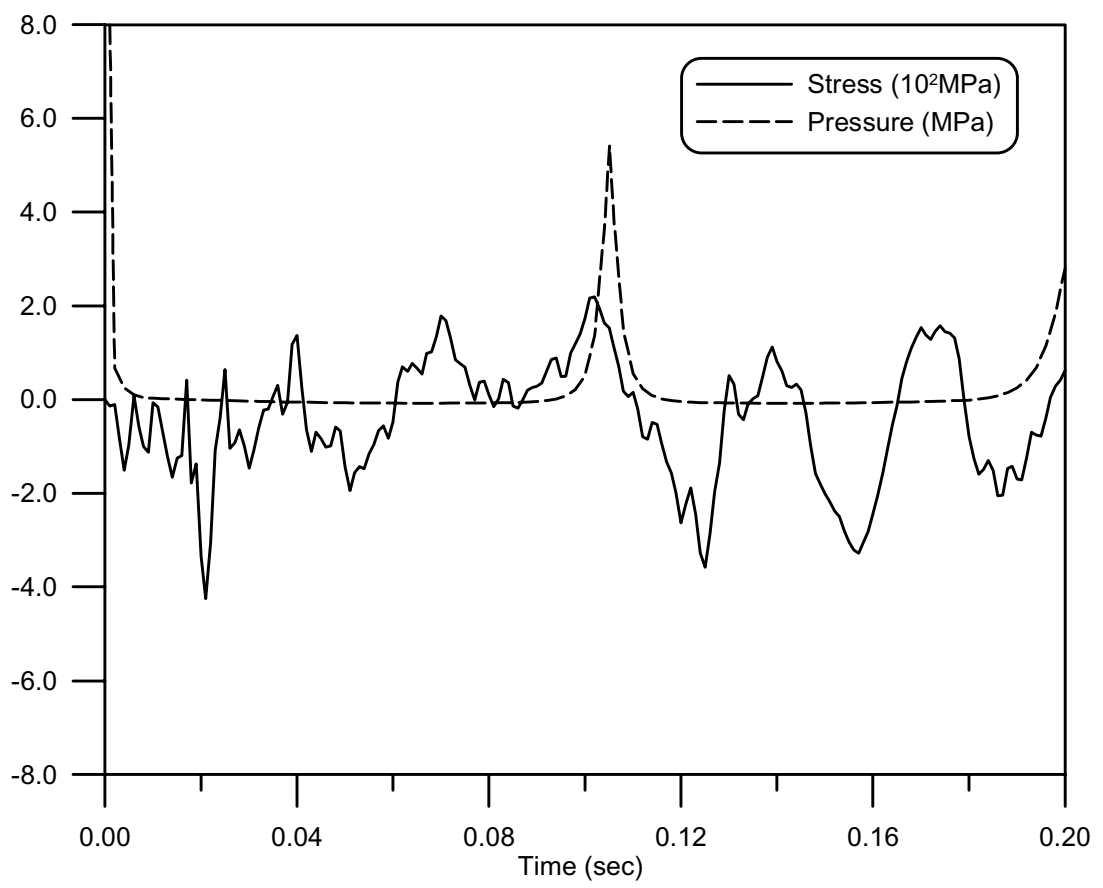

Fig. 9. Pressure and stress at the standoff point. 


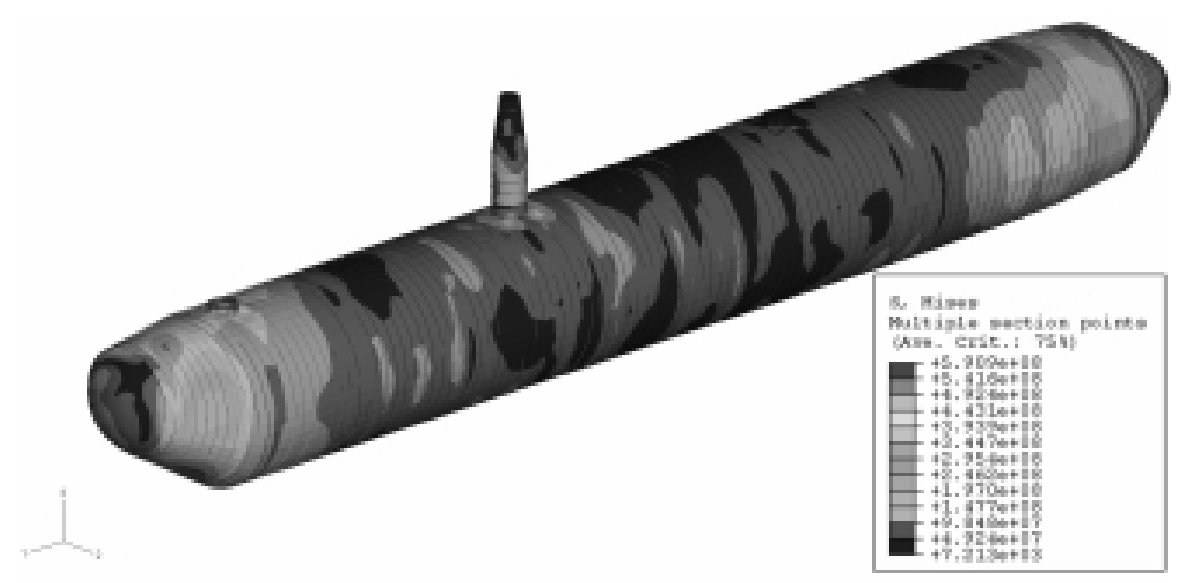

Fig. 10. Stress distribution at $t=0.01 \mathrm{sec}$.

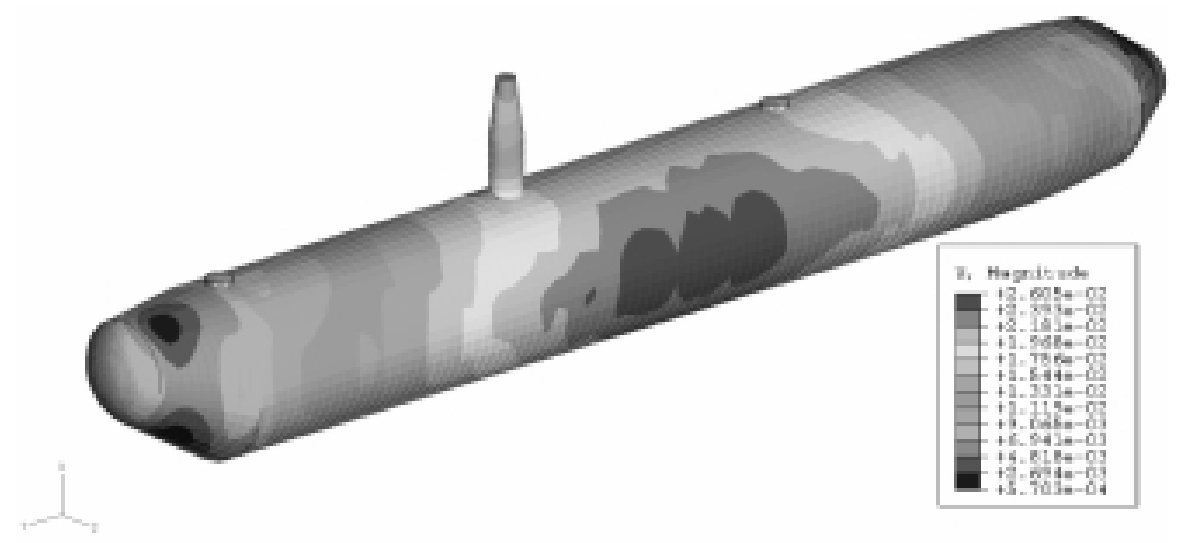

Fig. 11. Deformation distribution at $t=0.01 \mathrm{sec}$.

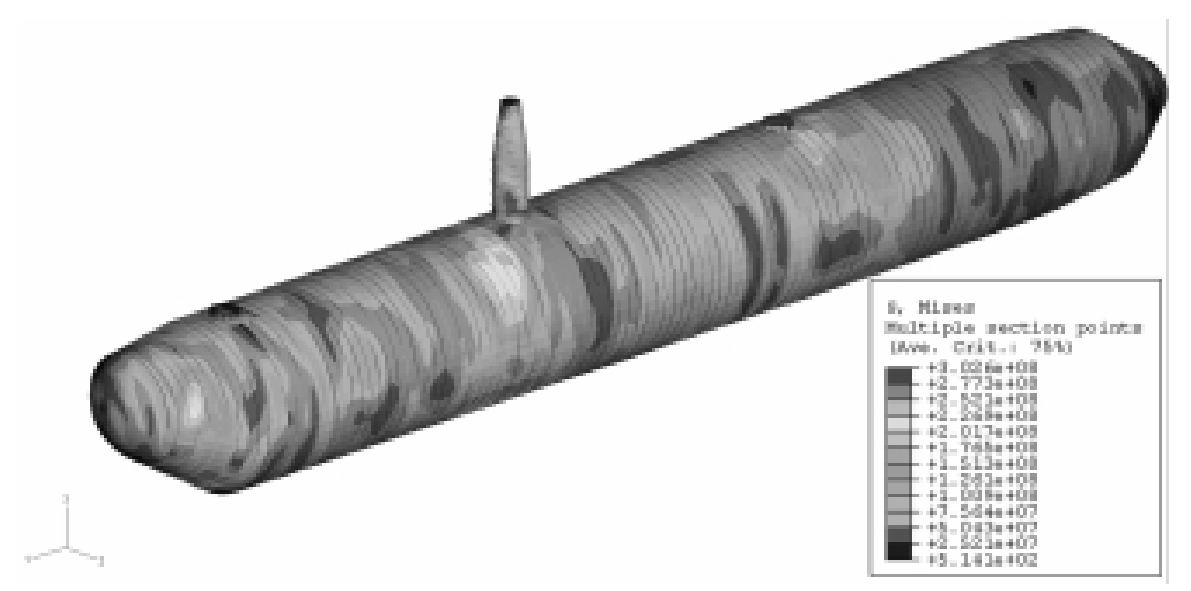

Fig. 12. Stress distribution at $t=0.04 \mathrm{sec}$. 


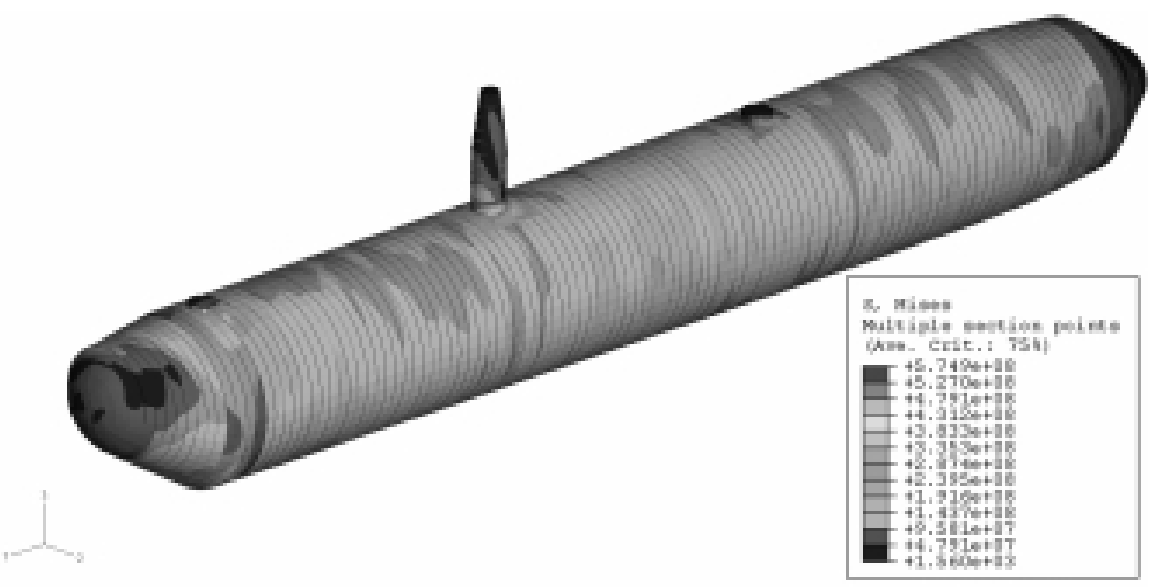

Fig. 13. Stress distribution at $t=0.12 \mathrm{sec}$.

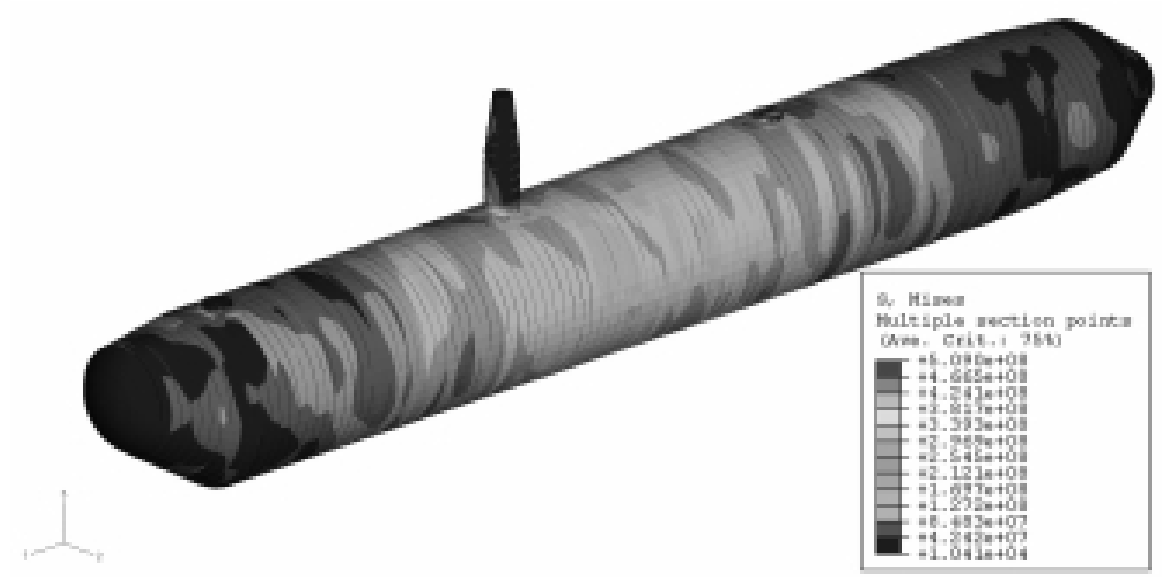

Fig. 14. Stress distribution at $t=0.20 \mathrm{sec}$.

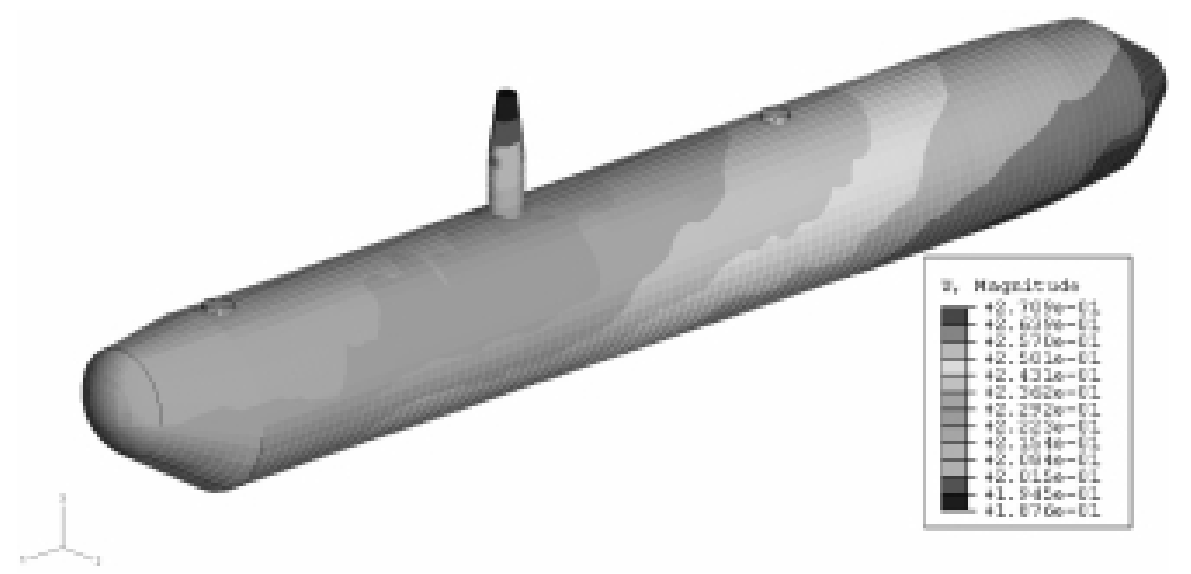

Fig. 15. Deformation distribution at $t=0.20 \mathrm{sec}$. 


\section{Conclusion}

(1) The FEM packages may be more mature and correct, and take more computing time than the theoretical method when solving for the dynamic bubble phenomenon. In this research, the theoretical method, which was applied to solve the pulsating dynamic bubble equation and the pressure variation, obtained a satisfactory solution compared with a FEM solution, and consumed less computer resources. This scheme is recommended for use during early design stages.

(2) Three bomb positions were tested: beside, below, and above the hull. When the bomb explodes below the submarine, it produces the most serious damage to the hull. When the bomb explodes above the hull, the damage is slighter than the other two cases.

\section{Acknowledgements}

The authors would like to thank the National Science Council of the Republic of China, Taiwan, for financially supporting this research under Contract No. NSC92-2611-E-002-017.

\section{References}

[1] J.S. Coles, E.A. Christian, J.P. Slifko, C.R. Niffennegger and M.A. Rogers, Shock wave parameters from spherical TNT charges detonated underwater, in: Underwater Explosion Research, Vol. 1, Office of Naval Research, Washington, DC, 1950.

[2] L. Rayleigh, On the Pressure Developed in a Liquid During the Collapse of a Spherical Cavity, Philosophical Mag 34 (1917), 94-98.

[3] H. Lamb, The Early Stages of a Submarine Explosion, Philosophical Mag 45 (1923), 257-265.

[4] H. Jones and A.R. Miller, The Detonation of Solid Explosives, Proceedings of the Royal Society of London, Series A, Mathematical and Physical Sciences 194 (1948), 480-507.

[5] R.M. Davies and G.I. Taylor, The Vertical Motion of a Spherical Bubble and the Pressure Surrounding It, in: Underwater Explosion Research, Vol. 2, Office of Naval Research, Washington, DC, 1950

[6] K.S. Hunter, Underwater Explosion Bubble Dynamics, B.S., New Mexico State University, 1993.

[7] M. Strasberg, Gas Bubbles as Source of Sound in Liquids, The Journal of the Acoustical Society of America 28 (1956), 20-26.

[8] R.H. Cole, Underwater Explosions, Princeton University Press, Princeton, 1948.

[9] C.E. Brennen, Cavitation and Bubble Dynamics, Oxford University Press, New York, 1995.

[10] C.N. Wang and C.C. Tse, The Scattering of an Acoustic Wave Incident on the Rigid Floating Body, Applied Acoustics 53 (1998), $35-41$.

[11] ABAQUS/Theory Manual Version 6.1-1, Hibbitt, Karlsson \& Sorensen Inc., 2000.

[12] Y. Ikeda and T. Katayama, Roll Damping Prediction Method for a High-Speed Planning Craft, Proceeding of the 7th International Conference on the Stability of Ship and Ocean Vehicles, B 2000, 532-541. 

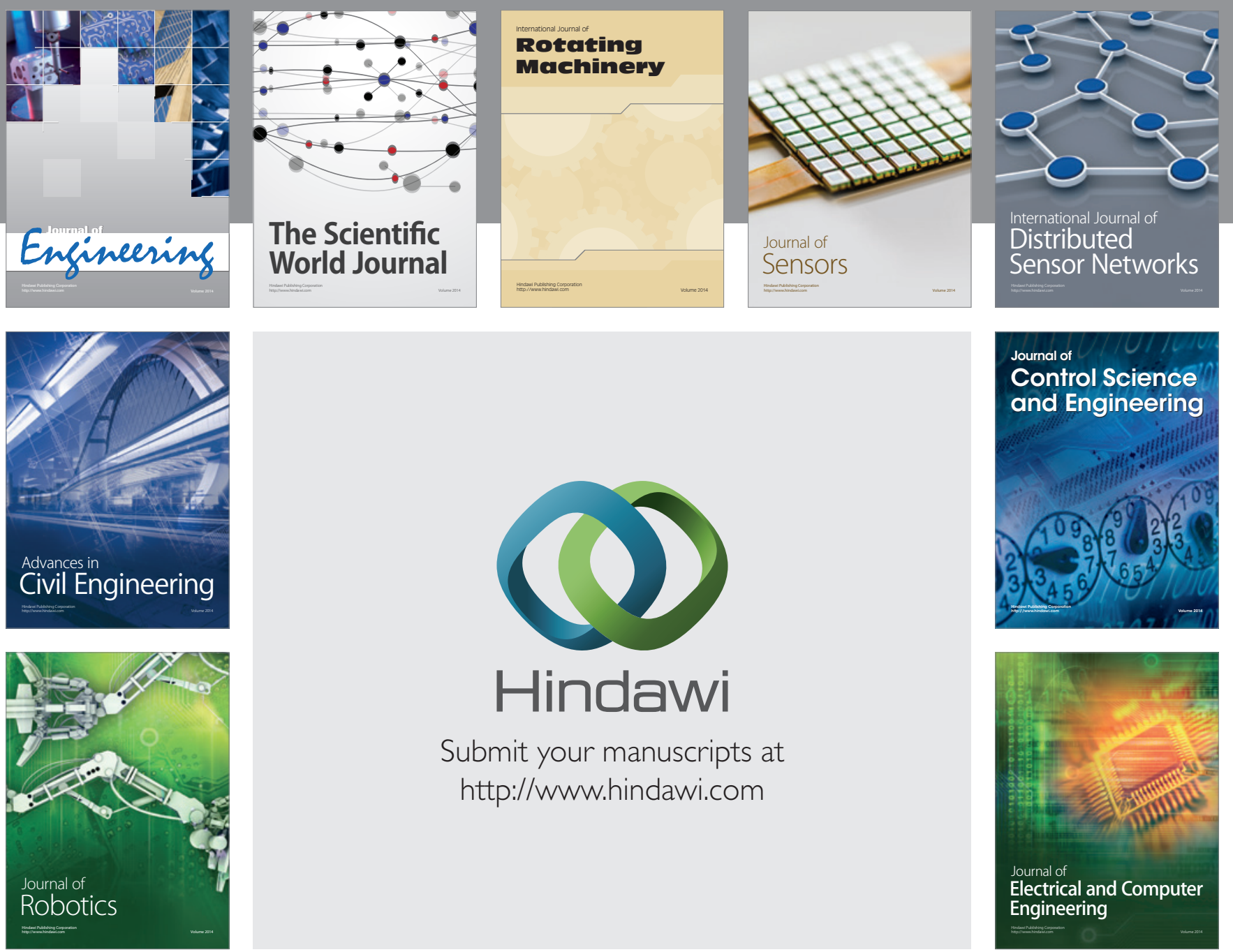

Submit your manuscripts at

http://www.hindawi.com
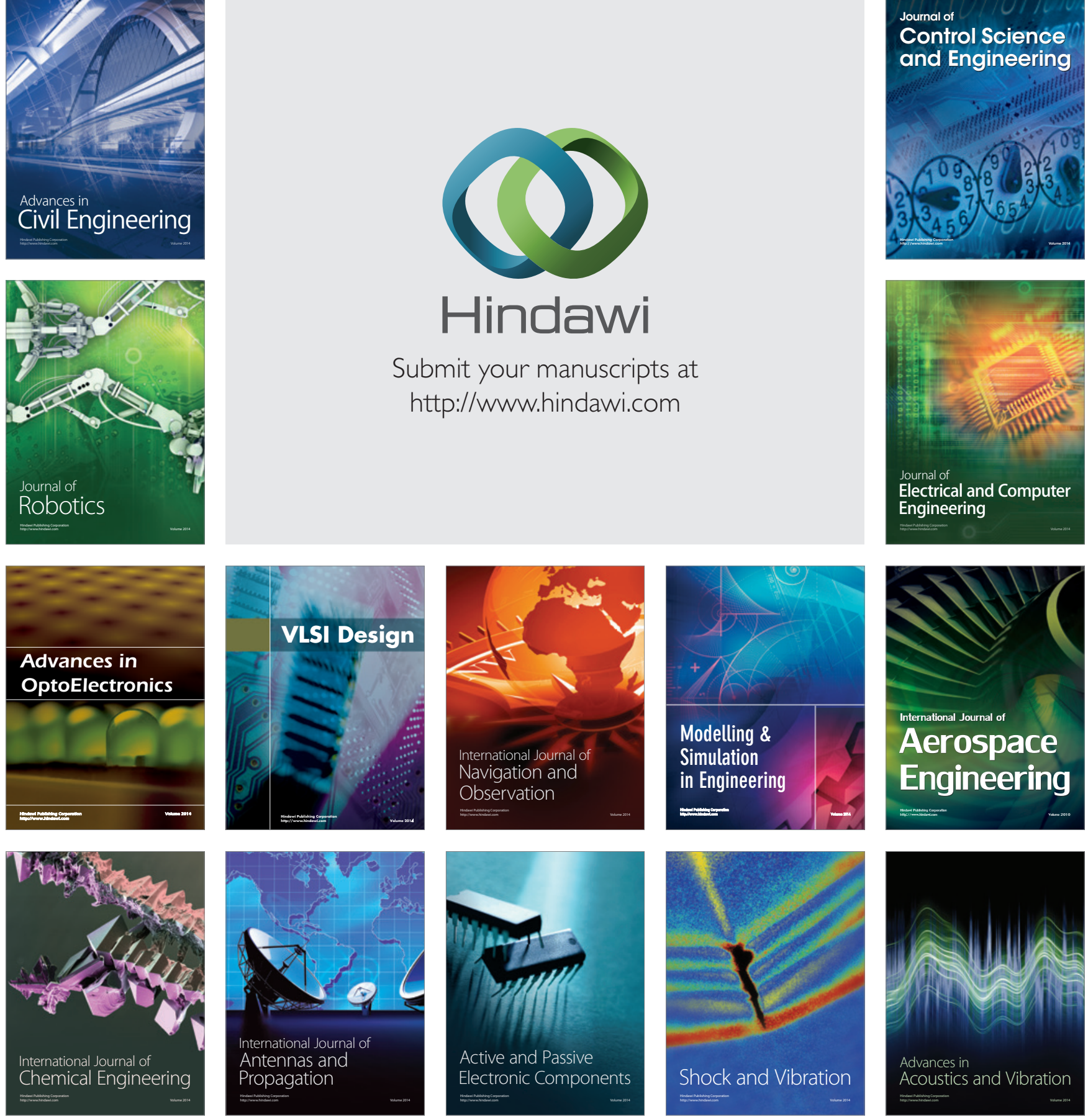